\title{
Current needs of the pharmaceutical industry: opportunities and challenges for implementing novel drying technologies
}

\author{
Langford, A. ${ }^{\text {a }}$ Luy, B. ${ }^{\text {b; Ohtake, S. }}{ }^{a^{*}}$ \\ ${ }^{a}$ Pfizer, Inc. BioTherapeutics Pharmaceutical Sciences, Chesterfield, MO, USA. \\ ${ }^{\mathrm{b}}$ Meridion Technologies GmbH, Müllheim, Germany. \\ *E-mail of the corresponding author: Satoshi.ohtake@pfizer.com
}

\begin{abstract}
Commercial drying methods are limited either by high production costs or significant quality loss due to process-related stresses. The near-ubiquitous use of freeze-drying in the pharmaceutical industry makes it the standard to which other drying technologies are compared. However, the shortcomings of lyophilization warrant evaluation of new techniques and the benefits they offer, such as compatibility with continuous manufacturing. Novel drying technologies must also overcome barriers to commercial implementation including, but not limited to, scalability and integration into a GMP environment. There remain several opportunities for further research which direct focus and investment strategy for the next generation pharmaceutical drying technologies.
\end{abstract}

Keywords: pharmaceuticals; manufacturing technology; implementation; lyophilization; scalability 
Current needs of the pharmaceutical industry: opportunities and challenges for implementing novel drying technologies

\section{Introduction}

The dehydration of wet material can enable convenient storage options, decrease transportation costs, and improve the ease of sample handling. The specific mechanism of water removal plays a significant role in whether these benefits are achieved since the drying process impacts the material's physical and chemical properties. A combination of consumer demands for improved functional properties (e.g., rapid dissolution, longer shelf life) and manufacturers' concerns with the financial and operational aspects of a drying process are major drivers for implementing novel drying technologies in industrial applications (Fig. 1). In the food industry, J. A. Moses and coauthors rightly point out that "with technology and market-driven demands, new dryers will continually be developed.”[1]

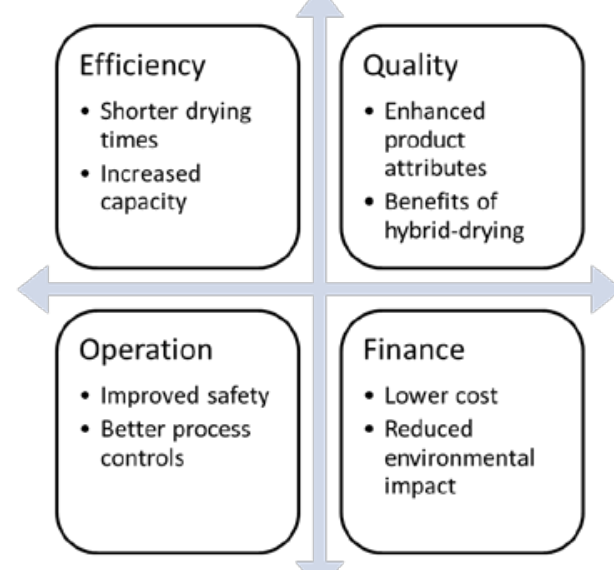

Fig. 1 Drivers for novel drying technologies. Figure adapted from Moses et al. [1]

It is critical for the pharmaceutical industry to have a similar mindset regarding drying technologies, especially since pharmaceuticals employ considerably fewer number of drying techniques during manufacture compared to foodstuffs. Additional drivers for the evaluation of new drying technologies include, but are not limited to, improved energy efficiency, reduced environmental impact (e.g., use of renewable energy sources), and compatibility with continuous manufacturing process. For these reasons, having a strategy for evaluating and implementing new manufacturing technologies is critical to meet the demands for reducing manufacturing costs and delivering next generation therapeutics.

\section{Challenges and opportunities for new technology implementation}

While we remain optimistic that the best scientific ideas will be appropriately nurtured, there are many obstacles to their growth, development, and future implementation. In simple terms, humans are often innately resistance to change. As we explore unknown areas, we move away from safety and comfort and into risk and potential frustration. Therefore, it is critical to keep an open mind when new technologies are being assessed. Management (in industry) should be wary to stifle an idea simply because it may not be 
easily implemented or scalable. Pushing through the initial resistance associated with developing a new manufacturing technology may lead to long-term benefits. Consumers (i.e., HCP and patients) may need to be re-educated to utilize products with 'atypical' features since novel drying technologies may produce products possessing unique physical properties (e.g., density, color), which may differ from those being produced by currentlyaccepted technology.

A biopharmaceutical technology management strategy outlined by Thakur et al. describes a four-phased approach to improving capabilities which involves observation, alignment, decision, and action.[2] Observation requires detailed collection of information and data from both internal and external sources. A market assessment conducted by Janssen summarized seven key technology themes that may impact the manufacturing process.[2] These technology themes include; process innovation, single use/modularity, continuous, automation/robotics, data analysis, disruptive, and customer facing. Technology trends impacting all aspects of biopharmaceutical processing, including upstream and downstream bioprocessing, formulation and fill, and packaging/devices, can be easily sorted into these overarching themes.[2]

In some ways, regulators are playing a leading role in the conversation on the implementation of new manufacturing technologies. A statement released in September 2017 describes the FDA's commitment to pharmaceutical innovation, not only in regards to novel therapeutics, but also in the manufacturing technologies used to produce them. [3] CDER's Office of Pharmaceutical Quality created an Emerging Technology program to "promote the adoption of innovative approaches to pharmaceutical manufacturing... and [provide] a route for companies to engage with the FDA prior to regulatory submission”.[3] In spite of these support structures in place, Munk [4] has summarized a number of challenges being encountered for implementation, including: 1) need for precedence, 2) need for a defined regulatory path, robust single-use technology, and robust process analytical technology (PAT) tools, 3) lack of comfort level and control tools, 4) lack of easy fit for continuous manufacture into existing infrastructure, facilities, and quality systems, 5) need for economic justification and adaptation of current quality or regulatory programs, and 6) need for unit operations to be fully developed for continuous processing.

While some of these concerns are specific to continuous manufacture, the core principles apply to the resistance shown and felt by the industry to accept the implementation of any new technology. There are further pharma-specific aspects for consideration. For legacy products, new technologies are not easily implemented; tremendous amounts of effort have been invested to fulfill the regulatory requirements of product approval and maintain the validated status. Often, the introduction of a new technology would represent a 'Major Change' that requires a very costly repetition of validation and requalification work, in addition to the risk that may be associated with the use of new technology.

Alternatively, a new technology may be introduced in conjunction with a new compound. This, however, narrows the range of potential users to introduce a new technology to the Rx-based industry. Furthermore, very significant risk is linked to the development of new compounds, which can fail in late development stages or even after market approval. To invest in a cGMP manufacturing site with a new technology that is not available in other sites of the company, or industry, for a new compound further increases the risk. 
Current needs of the pharmaceutical industry: opportunities and challenges for implementing novel drying technologies

\section{Challenges/limitations with lyophilization}

Lyophilization is the industry standard for drying of biopharmaceutical products and a significant fraction of pharmaceutical industry's knowledge is devoted specifically to freeze-drying.[5] Even though it is common-practice, there are several drawbacks to the lyophilization process, such as 1) high energy consumption, 2) long processing times, 3) significant production and maintenance cost, and 4) limited manufacturing and container closure flexibility.

The energy efficiency of a fully loaded production-scale lyophilizer was reported by Alina Alexeenko to be as low as 1.5 to 2\%.[6] In general, drying processes are one of the most energy intensive unit operations.[7] The long drying process times during freeze-drying, which can vary from several hours to days, may also limit the number of batches that can be produced at manufacturing site. In some cases, the lyophilizer capacity may be the limiting factor to overall manufacturing production rate. This is one of the main reasons that significant effort is focused on optimizing the primary and secondary drying times during the freeze-drying cycle.[8] Since lyophilization is a batch process, it will also be challenging to adapt the technology into a continuous manufacturing process. From a container closure perspective, as previously mentioned, the primary packaging is limited to containers that are suitable for lyophilization (e.g. vial or bottles).

Despite these limitations, freeze-drying is a robust and scalable process that is widely applied in the pharmaceutical industry. In all likelihood, freeze-drying will remain the predominant method for drying pharmaceuticals; however, the drawbacks highlighted here demonstrate the potential benefit of evaluating next generation drying technologies that may overcome these challenges.

\section{Potential solution to limitations associated with lyophilization}

In alignment with the technology management strategy, spray-freeze drying is a unique drying technology that is being evaluated for biopharmaceuticals due to the plethora of its offerings, which among them include manufacturing process innovation and new packaging options for customers [9-11]. Spray freeze-drying technology can be aimed at two major focus areas: product innovation and increased manufacturing flexibility. With respect to manufacturing flexibility, a major aspect is the production of homogeneous, free flowing and sterile microspheres as bulk that can be filled into vials, or any other container closure systems, at any time, with flexible (vial) dosing and batch quantities (e.g. even personalized medication). In contrast to conventional vial lyophilization, the general sequence of operation is reversed by having the lyophilization process prior to the filling process. With this technology, a long and rigid supply chain can be made flexible and cold chain logistics can be facilitated.

Since the product is already freeze-dried, the primary packaging does not need to be suitable for lyophilization; this allows for direct filling into syringes or any other application devices that would not be suitable for conventional shelf freeze-drying. 
With respect to product innovation, the availability of free flowing bulk that can be dosed and filled allows to formulate combination products by compounding on the filling line. Furthermore, the availability of the increased product surface not only facilitates the drying due to increased heat and mass transfer, but also reduces reconstitution time. This specifically applies when high protein concentration products (up to $200 \mathrm{mg} / \mathrm{ml}$ ) are processed. Spray freeze-drying is a good example of a technology that has successfully progressed through the technology management process and will continue to mature as more applications are investigated.

As described above, the introduction of new, disruptive technologies in pharmaceutical applications has its challenges, which has an impact on the overall product development timeline that needs to be taken into account (i.e., from first process development studies to cGMP operations). This can easily cover a period of $10+$ years. This time span is a challenge even for an established technology provider, as the return on investment for the development does not occur prior to broader implementation in the industry, and the profitability is not in the range of new pharmaceutical compounds. The risks and challenges are greater for start-up companies that need to survive economically in that long development period.

Nevertheless, there are possibilities to balance these risks. It is advisable to team up with a major Big Pharma partner that, besides the economical strength and possible application, brings regulatory competence and IP strengths to the development process. At a certain level of maturation, it may be possible to look for additional companies, which could then form a User Group, or a consortium, in order to address the development needs that are associated with the implementation of a new technology as well as regulatory considerations. In regard to the latter, it may be preferable to solicit participation of regulatory bodies that can comment on their evaluation criteria, which will reduce the risk of surprises regarding the acceptability of the technology.

Alternatively, it may be possible to look for applications outside of pharma that do not carry the intrinsic risks and other time-consuming formal evaluation as in pharma applications. In these sectors, faster development and evaluation can be accomplished. Even with this approach, the challenge still remains to progress developments that are specific to the pharmaceutical application in parallel to reduce the time associated with implementation.

\section{Conclusions}

While the pharmaceutical industry continues to demonstrate its creativity associated with novel compounds in development (now venturing into the realms of gene and cell therapy, while a decade ago, it was solely focused on monoclonal antibodies), the processing technology has not kept its pace. This is not a reflection of the paucity of innovation associated with processing technology. Regulatory aspects, in which conventional testing or validation procedures and routines that are well accepted may no longer be applicable, pose another challenge that pharma companies face when considering the introduction of new processes. The introduction of a disruptive new technology is often only possible if a promising new compound strongly requires the benefits of that new technology, and if the 
Current needs of the pharmaceutical industry: opportunities and challenges for implementing novel drying technologies

business case allows for the additional costs that are linked to its introduction. These concerns can broadly be classified as economic, logistical, technical, and psychological and all elements need to be overcome in order for successful implementation of a new technology.

\section{References}

[1] Moses, J. A.; Norton, Tomás; Alagusundaram, K.; Tiwari, B. K. Novel drying techniques for the food industry. Food Engineering Reviews 2014. 6 (3):43-55.

[2] Thakur, R.; Simmen, T.; Merkle, S.; Venkataraman, R.; Handor, C.; Van den Heuvel, R. Evaluating technology and innovation in biopharmaceutical manufacturing. Pharmaceutical Technology 2016. 40 (8):22-27.

[3] FDA in Brief: FDA issues guidance to help advance novel technology to improve the reliability and safety and help lower the cost of pharmaceutical manufacturing. 2017.

[4] Munk, M. 2017. "The industry's hesitation to adopt continuous bioprocessing: recommendations for deciding what, where, and when to implement." BioProcess International.

[5] Pikal, Michael J. Freeze Drying. In Encyclopedia of Pharmaceutical Technology; Swarbrick, James, Eds.; Informa Healthcare USA, Inc., 2006; pp 1807-1833.

[6] Alexeenko, A. 2011. "Controlling the freeze-drying process: Simulations and modeling." World Lyophilization Summit, Boston, Massachusetts.

[7] Raghavan, G. S. V.; Rennie, T. J.; Sunjka, P. S.; Orsat, V.; Phaphuangwittayakul, W.; Terdtoon, P. Overview of new techniques for drying biological materials with emphasis on energy aspects. Brazilian Journal of Chemical Engineering 2005. 22 (2):195-201.

[8] Tchessalov, S.; Dixon, D.; Warne, N. 2018. Lyophilization above collapse. US9884019B2.

[9] Luy, B.; Plitzko, M.; Struschka, M. 2016. Process line for the production of freeze-dried particles. EP2764309.

[10] Struschka, M.; Plitzko, M.; Gebhard, T.; Luy, B. 2016. Rotary drum for use in a vacuum freeze-dryer. EP2764310.

[11] Plitzko, M.; Struschka, M.; Gebhard, T.; Luy, B. 2015. A process line for the production of freeze-dried particles. EP2764311. 